\title{
Etnomotricidad: juegos de resistencia cultural en la comunidad caizara de Ilhabela - Brasil ${ }^{*}$
}

\author{
Ethnomotricity: cultural resistance games in community of caizara de Ilhabela - Brazil \\ Etnomotricidade: jogos de resistência na comunidade caiçara de Ilhabela - Brasil
}

\author{
Luiz Gonçalves Junior, Denise Aparecida Corrêa, Clayton da Silva Carmo, Silmara \\ Elena Alves de Campos, Sergio Alejandro Toro-Arévalo ${ }^{\text {a }}$
}

aUniversidade Federal de São Carlos, Brasil. Departamento de Educação Física e Motricidade Humana. Telf: +55 16 3351-8769. Correo electrónico: luiz@ufscar.br

\begin{abstract}
RESUMEN
Entendemos la Educación Física como uno de los componentes curriculares que puede y debe contribuir a la presentación del diálogo acerca de la diversidad cultural, razón por la cual proponemos el estudio de la etnomotricidad orientada a las manifestaciones relacionadas con juegos, luchas, danzas, fiestas, cantos y cuentos, característicos de un pueblo / comunidad desarrolladas con intencionalidad y asociadas a procesos educativos de tradición y resistencia cultural. Específicamente en este estudio, se pretendió caracterizar y comprender la etnomotricidad caizara intentando la comprensión de las prácticas y los procesos educativos en ellas involucrados, particularmente, junto a la comunidad caizara considerada tradicional (Playa Mansa y alrededores). Utilizando metodología cualitativa de inspiración fenomenológica, después de la inserción y consentimiento formal de la comunidad, se realizó el registro sistemático de los datos a partir de diarios de campo. A partir del análisis de las unidades de significado emergieron cuatro categorías: a) Desconfianza aprendida; b) Maritorio lúdico; c) Convivencia emergente; y d) Festividad religiosa. Consideramos que los resultados en esta investigación pueden contribuir con propuestas didácticas en el ámbito de la educación física, específicamente en el nivel de la educación básica, para el conocimiento y reconocimiento de prácticas de otros culturas o grupos étnicos minoritarios.
\end{abstract}

Palabras clave: etnomotricidad, motricidad humana, diversidad cultural, cultura caizara.

\begin{abstract}
Understanding the physical education as one of the curriculum components that can and it should contribute to the dialogue concerning cultural diversity we propose the study of the ethno-motricity, understanding it as manifestations related to the games, fights, dances, parties, songs and stories, characteristics of the people / community developed with intentionality and related to educational processes of tradition and resistance of such manifestations. Specifically in that study, we aimed to characterize and to understand the ethno-motricity inhabitant of the seaside looking at the understanding of the practices and the educational processes in them involved, particularly, close to the community inhabitants of the seaside considered traditional, of Tame Beach and adjacencies. Using qualitative methodology of phenomenology inspiration, after insertion and the community's formal consent, we accomplished systematic registration of data in field diaries. Ruled in the analysis of the units of meaning four categories emerged: A) Learned distrust; B) Play-oriented category which we name "Maritorio"; C) Emergent coexistence; D) Religious festivity. We considered that the results reached in this research can contribute with proposed didacticisms in the ambit of the physical education, especially in the basic education, for the knowledge and the recognition of practices and manifestations of other cultures or minority ethnic groups.
\end{abstract}

Key words: etno-motricity, human Motricity, culture inhabitant of the Seaside, cultural diversity.

* Este artículo fue solicitado por la Revista Estudios Pedagógicos en diciembre del 2011 en el contexto del proyecto de Investigación FONDECYT (Fondo de Investigación Científica y Tecnológica) No 11110016 , titulado "Educación Física y su función de transformación de las desigualdades sociales: profesorado del área y documentación ministerial”. El artículo fue aceptado en junio de 2012. 


\begin{abstract}
RESUMO
Entendendo a Educação Física como um dos componentes curriculares que pode e deve contribuir para a apresentação do diálogo sobre a diversidade cultural, propõe-se o estudo da etnomotricidade, cuja compreensão se volta para manifestações relacionadas a jogos, lutas, danças, festas, cantos e contos característicos de um povo / comunidade desenvolvida com intencionalidade e associada a processos educativos de tradição e resistência cultural. Pretendese caracterizar e compreender a etnomotricidade caiçara visando à compreensão das práticas e processos educativos nela envolvidos, particularmente aquelas relacionadas à comunidade tradicional caiçara de Praia Mansa e seu entorno. Utiliza-se metodologia qualitativa, de inspiração fenomenológica, e registra-se sistematicamente, após consentimento formal da comunidade, dados a partir de diários de campo. Das análises das unidades de significado, são criadas quatro categorias: a) desconfiança aprendida, b) maritorio lúdico c) convicência emergente e d) festividade religiosa. Acreditase que os resultados desta pesquisa possam contribuir com propostas educacionais no campo da Educação Física, sobretudo na Educação Básica, para o conhecimento e reconhecimento de práticas e manifestações de outras culturas ou grupos étnicos minoritários.
\end{abstract}

Palavras chave: etnomotricidade, motricidade humana, diversidade cultural, cultura caiçara.

\title{
1. INTRODUCCIÓN
}

En las clases de educación física ${ }^{1}$ escolar, comúnmente observamos el deporte como un contenido prácticamente exclusivo, lo que acaba por reducir el universo de la Motricidad Humana, circunscribiéndolo, no es de extrañar, al contexto cultural estadounidense y/o europeo del fútbol, vóleibol, básquetbol y hándbol, en detrimento de potencialidades que pueden ser exploradas al proponer la vivencia de otras manifestaciones de la Motricidad Humana (juegos, luchas, danzas, fiestas, cantos y cuentos), oriundas de la diversidad cultural de diferentes pueblos que construyeron y construyen el Brasil, tales como los africanos, los indígenas y los orientales (Gonçalves Junior, 2007).

Comprendemos, con Freire (2005: 156), que no se trata de "yuxtaposición de culturas, mucho menos en el poder exacerbado de una sobre las otras, sino en la libertad conquistada, en el derecho asegurado de moverse cada cultura en respeto de la otra, corriendo el riesgo libremente de ser diferente, de ser cada una para sî́”.

En este sentido, los Parámetros Curriculares Nacionales-PCNs (Ministério da Educação e do Desporto, Gobierno Federal de Brasil, 1997: 7) indican la importancia de "conocer y valorizar la pluralidad del patrimonio sociocultural brasilero, bien como aspectos socioculturales de otros pueblos y naciones, posicionándose en contra de cualquier discriminación basada en diferencias culturales, de clase social, creencia, sexo, etnia o características individuales y sociales".

El mismo documento indica:

La Educación Física permite que se vivencien diferentes prácticas corporales provenientes de las más diversas manifestaciones culturales y se observe como esa variada combinación de influencias está presente en la vida cotidiana. Las danzas, deportes, luchas, juegos y gimnásticas componen un vasto patrimonio cultural que debe ser valorado, conocido y disfrutado. Además de eso, ese conocimiento contribuye para la adopción de una postura no prejuiciosa y discriminatoria frente a las manifestaciones y expresiones de los diferentes grupos étnicos y sociales y de las personas que de ellos hacen parte (Cit. Ministério da Educação e do Desporto, 1997: 28-29).

1 Manuel Sergio (1994), uno de los principales pensadores de la Ciencia de la Motricidad Humana, prefiere el uso de la expresión Educación Motora en lugar de Educación Física. Nosotros preferimos Motricidad Escolar, no obstante, en esta investigación no haremos diferenciación entre una expresión y la otra, pues se considera la educación física la nomenclatura oficial del respectivo componente curricular. 
Frente a esta situación y entendiendo la Educación Física como uno de los componentes curriculares que puede y debe contribuir a la presentación, diálogo, reflexión y valorización de la diversidad cultural, proponemos el estudio de la Etnomotricidad, conceptualizando ésta "como prácticas corporales (juegos, actividades lúdicas, luchas, danzas) con características propias de un pueblo/comunidad, desarrolladas con intencionalidad ${ }^{2}$ y relacionadas a procesos educativos de tradición y resistencia de tales manifestaciones" (Gonçalves Junior, 2010: 51).

Explicitamos que la etnomotricidad se ancla en la ciencia de la motricidad humana de Manuel Sergio y en la pedagogía dialógica de Paulo Freire, las cuales, entre otras influencias, tienen base en la fenomenología existencial de Maurice Merleau-Ponty (Gonçalves Junior, 2010).

Consideramos que en la perspectiva fenomenológica se entiende al ser en el mundo a partir de los existenciales básicos, a saber: afectividad, comprensión y expresión, que están siempre en un mismo nivel de importancia, siendo, por tanto, equi-primordiales. De esta manera, son fundantes de la constitución del ser: son modos del existir-ahí. Existiendo-ahí-en-el-mundo, el ser se encuentra en una condición de abertura a la experiencia y, en esa abertura, "no hay un sentir anterior o posterior al comprender y expresar y vice-versa. El hombre que siente es al mismo tiempo el que comprende y expresa. Al expresarse, el hombre abre espacios de comprensión, y toda comprensión, en sí, ya es afectiva" (Silva, 1991: 8).

En palabras de Merleau-Ponty (1996: 235), un orador no piensa antes de hablar, incluso mientras habla; su hablar es su pensamiento, el pensamiento no existe fuera del mundo o para sí antes de la expresión; ni siquiera la pretensión de silencio susurrando es el hablar. Del mismo modo, no es preciso que el ser represente el espacio exterior y su cuerpo para mover uno de otro; basta que ellos existan para el ser, pues "todas las funciones" en el hombre, desde la sexualidad a la motricidad y a la inteligencia, son rigurosamente solidarias; es imposible distinguir, en el ser total de lo humano, una organización corporal que tratásemos como un hecho contingente, y otros predicados le pertenecerían con necesidad. Todo es necesario en el hombre."

Entonces, de acuerdo con Merleau-Ponty:

(...) la conciencia se proyecta en un mundo físico y tiene un cuerpo, así como ella se proyecta en un mundo cultural y tiene hábitos: porque ella sólo puede ser consciencia jugando con significaciones dadas en el pasado de la naturaleza o en su pasado personal (...). En fin, esas claridades nos permiten comprender sin equívoco la motricidad en cuanto intencionalidad original. (...) El movimiento no es el pensamiento de un movimiento, el espacio corporal no es un espacio pensado o representado (...) la motricidad no es como una sierva de la conciencia que transporta el cuerpo al punto del espacio que previamente nos hemos representado. Para que podamos mover nuestro cuerpo en dirección a un objeto, primeramente es preciso que el objeto exista para él (Merleau-Ponty, 1996: 192-193).

La motricidad humana, conforme lo dicho por Sérgio, se trata de un "movimiento intencional de la trascendencia, es decir, el movimiento de significación mas profunda" (Sérgio, 1999: 17) en el que lo esencial "es la experiencia originaria, donde emerge también la historia de las conductas motoras del sujeto, pues no hay experiencia vivida sin la

2 Se refiere la intencionalidad de comportamiento corpóreo-mundano (existencial), en la cual se construye y reconstruye el mundo significado, pues el encuentro de consciencia y mundo es el origen de ambos (Fiori, 1986). 
intesubjetividad que la praxis supone. El ser humano está todo en la motricidad, en una continua abertura hacia la realidad más radical de la vida" (Cit. en Sérgio, 1999: 17-18). Aún mas, el autor agrega que "la motricidad humana significa que el ser humano es fundamentalmente relación con el Otro, con el Mundo, con lo Absoluto" (Sérgio, 1994: 71).

En tal perspectiva el ser es comprendido en su integralidad existiendo-ahí-en-el-mundocon-los-otros, dotado de la posibilidad de transcendencia (de ser más), en condición de abertura para la experiencia y, en esa abertura, no hay posibilidad de fragmentación. De modo que al chutear el balón, por ejemplo, se está impregnado de experiencia de ser en el mundo, es decir, cargado de emoción, de sentimiento, de su cultura y de intencionalidad. Por lo tanto, ese gesto tan común en nuestros días está muy lejos de ser un mero acto mecánico restrictivo y muy cerca de una acción compleja de constituir mundo al mismo tiempo que constituir identidad.

Como escriben Martins y Machado (1997), el comportamiento humano no es una serie de reacciones ciegas a los estímulos, ni es la proyección de actos motivados por la idea pura producida por la mente desencarnada y sin mundo; más bien es un interrelacionamiento dialéctico y dialógico entre el ser y el mundo que no puede ser expresado en términos de causalidad.

Desde esta matriz, ampliamos el marco de análisis para adentrarnos en la Etnomotricidad, entendida desde la particularidad que constituye un grupo humano en una determinada localización espacio-temporal, en una dinámica relacional, simbólica y funcional singular. En otras palabras, podemos entender la etnomotricidad como ese estar ahí en el mundo de un grupo de seres humanos que les otorga identidad, historia, proyección diferenciada. Por lo cual, no es sólo conductas diferentes que pueden manifestar en relación con un determinado marco dominante, sino más bien es una percepción radical de mundo diferenciada que favorece determinadas comprensiones e interpretaciones de la acción humana, de su sentido y habitabilidad en el mundo. En consecuencia sólo sería posible compreder las acciones de un grupo social si nos adentramos profundamente en su mundo simbólico y comprensivo de su día a día y cara a cara, como nos propone Schutz (1995). De manera que juegos, luchas, danzas, fiestas, cantos y cuentos se configuran en forma particular tanto en su ejecución como en su comprensión, lo que también podría considerar, obviamente, diferenciaciones morfo-funcionales.

En esta investigación, en particular, nos centramos en la Etnomotricidad Caizara, específicamente de la comunidad de Playa Mansa y alrededores (playa de los Castellanos y Playa Roja), que corresponden al Municipio de Ilhabela, litoral norte del estado de San Pablo. Explicitamos también que Caizara es un término proveniente de la lengua indígena tupi, que denominaba originariamente las ramas de árboles colocados en el mar para cercar los peces en una jaula. Posteriormente pasó a ser utilizado, también, por los habitantes y comunidades de zonas litorales de los estados de São Paulo, Rio de Janeiro y Paraná, los cuales, en el pasado, tenían un modo de vida exclusivamente ligado a la pesca y la agricultura. Las comunidades caizaras nacieron a partir del siglo XVI, produto del mestizaje de blancos (principalmente portugueses), indígenas (tupinambás) y negros (transportados como esclavos desde de Africa al Brasil) (Cfr. Principalmente Adams, 2000; Diegues, 2005; Campos, 2008).

Esta investigación pretende contribuir al fortalecimiento de la identidad de grupos/ etnias/culturas socialmente marginados, favoreciendo el respeto por la diversidad cultural y el medio ambiente; y también apoyar a profesores de educación básica en la 
formación de escolares, promoviendo el conocimiento y respeto sobre la motricidad de diferentes culturas.

\section{ANTECEDENTES GENERALES DEL MUNICIPIOY LOS HABITANTES DE ILHABELA}

Partiremos con una descripción general de la isla a fin de comprender con mayor claridad tanto la formulación del objetivo, como el trabajo desarrollado en Ilhabela. Dicha Isla se encuentra localizada en el litoral norte paulista, a 230 kilómetros de la ciudad de San Pablo y, en realidad, es un archipiélago compuesto por las islas de: San Sebastián, Victoria, de Búzios, de las Cabras, Sumítica y de Serraria, teniendo la mayor concentración poblacional en el área urbanizada de la Isla de San Sebastián. En estas última se encuentran las playas más frecuentadas por los turistas: Curral, Armazon e Feiticeira (Campos, 2008).

Desde el punto de vista de su geografía humana y de acuerdo con Diegues (2005), la población caizara de dicha región está íntimamente ligada a la Selva Atlántica, ${ }^{3}$ pues de ella obtienen la madera utilizada en la construcción de canoas y otros artefactos, también practican la extracción de frutos y hiervas y la agricultura de alimentos y plantas medicinales.

Por otra parte, conforme lo presentado por Corrêa (1981), los caizaras provienen de antiguos cruces entre indígenas brasileros y colonizadores europeos y, en la época de la esclavitud, de nuevas combinaciones étnicas con el contingente de esclavos africanos quienes tuvieron una importante participación no sólo en términos numéricos, sino también culturales en la conformación actual de la cultura caizara. Específicamente, dicho autor sostiene que "Predominan caracteres del colonizador blanco (...). En la sociedad local, la religión, los hábitos y las costumbres son del colono europeo de los primeros siglos del conquista brasilera, cuyas características, principalmente culturales se conservan" (Corrêa, 1981: 19).

Actualmente, motivados por el crecimiento del turismo, el desplazamiento de los caizaras desde el borde costero hacia el interior de la isla, junto a las leyes ambientales que les prohíben cortar árboles para la producción de canoas o la delimitación de una cuota de árboles. Al mismo tiempo, en la misma región se han desforestado grandes extensiones de Mata Atlántica para la construcción de mansiones de veraneo, lo que ha potenciado el desplazamiento de los caizaras y el consecuente dificultad para mantener su modo de vida original, especialmente en las regiones más urbanizadas del archipiélago (Campos, 2008).

Según Villela (2003), con la llegada de la actividad turística en la ciudad, las nuevas relaciones introducidas afectaron profundamente al caizara, provocando su dislocamiento físico y social. Los caizaras pasaron a instalarse en lugares más separados de la playa y del centro, perdiendo sus zonas originales. Las comunidades que, de alguna manera, mantuvieron su cultura, son las que se encuentran en lugares de mayor dificultad de acceso, las cuales son denominadas comunidades caizaras tradicionales.

La denominación en portugués es "Mata Atlântica" y corresponde a la vegetación de selva tropical lluviosa, abundante en especies vegetales y animales. Este tipo de vegetación abarcaba toda la costa del Brasil, bañada por el océano Atlántico en la época de la conquista; actualmente se encuentra reducida a menos de la mitad. 
El proceso anterior se entiende mejor si se considera que, según Diegues, la tradición se entiende como un "proceso histórico por el cual elementos de la cultura llamada moderna son continuamente reinterpretados e incorporados al modo de vida" (Diegues, 2005: 275) y que la cultura caizara desarrolló un conjunto de prácticas materiales e inmateriales, valores, visones de mundo ligadas al mismo tiempo al mar y a la tierra. En sus propias palabras, es definida como:

(...) un conjunto de valores, visiones de mundo, prácticas cognitivas y símbolos compartidos, que orientan a los individuos en sus relaciones con la naturaleza y con los otros miembros de la sociedad y que se expresan también en productos materiales (tipos de moradas, embarcaciones, instrumentos de trabajo) y no materiales (lenguaje, música, danza, rituales religiosos) (Diegues, 2005: 275).

Vale destacar que los primeros puntos de poblamiento europeo en Brasil acontecieron en la región litoral y sólo más tarde ocurrieron procesos de colonización hacia el interior del territorio. Este aspecto resulta relevante si se considera que Diegues (2005: 282) especifica que la población caizara es originaria del indígena, del colonizador portugués (en algunas áreas, también español) y, en menor grado, del esclavo africano. Tal población, dispersa en la zona costera, estuvo ligada a los ciclos económicos, generalmente marcados por la monocultural agrícola, abasteciendo de productos de primera necesidad, como harina de mandioca, pescado y leña, a los núcleos urbanos regionales. El autor parte de la hipótesis que las poblaciones y los sitios caizaras surgieron en los intersticios y en el periodo de la pos-desorganización de las monoculturas coloniales y pos-coloniales como el de la caña de azúcar, en el litoral sur de Río de Janeiro y el norte de San Pablo:

La formación de las comunidades caizaras está, sin duda, asociada a una vuelta de parte de la población a las actividades agrícolas de casi subsitencia y hacia la pequeña pesca, pues el final de cada ciclo económico significó, en muchos casos, la vuelta de actividades con relaciones tenues con el mercado, como la micro-agricultura y pesca, marcando la marginalización de la población local (Diegues, 2005: 282).

Por otra parte, Almeida nos dice que "si se estudia la genealogía de las familias caizaras de las ciudades históricas, se observará que son, generalmente, descendientes directos de los fundadores de esas localidades y que aún conservan las tradiciones de familia, historia, usos y costumbres de sus ancestros" (2005: 48). La posesión de sus tierras les fue otorgada por cartas de sesmaria -cartas de posesión de tierras entregadas en la época imperial del Brasil- entregadas a los primeros habitantes y fueron sucedidas a los hijos sucesiva e ininterrumpidamente. Tal situación provoca que los caizaras tengan su historia de vida y familiar fuertemente relacionada con el espacio territorial y con un gran apego a "...propiedades que recibieron por herencia, y que parecen hacer parte de su yo, transformadas en pedazos de su alma, y de las cuales difícilmente se separan, conservándolas religiosamente" (Almeida, 2005: 48).

La Selva Atlántica fue de extrema importancia para los caizaras en la primera mitad del siglo XX, pues de ella era retirada la madera utilizada en la construcción de canoas, casas, muebles, utensilios domésticos, equipamientos de pesca, instrumentos musicales. Además, en ella se implementaban los métodos de caza, como la ceva. ${ }^{4}$ Aún en la ac-

$4 \quad$ Lugar donde se colocan los alimentos para atraer pesca o caza. 
tualidad, los caizaras mantienen fuerte relación con la Selva Atlántica, donde hacen la agricultura itinerante para la extransión de diversos productos (Diegues, 2005).

Almeida (2005) critica a los autores de su época que caracterizan a los caizaras como despreciables y vagabundos incorregibles. Y, en su parecer, más bien se caracterizan como un pueblo hospitalario. Cualidad heredada de los antiguos paulistas, y que debido al aislamiento en que viven, forman una casta diferenciada, en la cual no es fuerte el cosmopolitismo de las grandes ciudades y poblaciones, conservando los usos, costumbres y tradiciones de sus antepasados.

Para Diegues, en el territorio caizara "se desarrolló un modo de vida basado en la pequeña producción de mercaderías, que asocia a la pequeña agricultura y a la pesca, además de elementos culturales comunes, como el lenguaje característico, fiestas y una forma específica de ver el mundo" (Diegues, 2005: 276).

Buscando estudiar la etnomotricidad caizara, se realizó una inserción en la comunidad de Playa Mansa y alrededores (Playa de los Castellanos y Playa Roja), localizada en la parte no urbanizada de Ilhabela y considerada como una comunidad tradicional.

\section{PROCEDIMIENTOS}

Se partió de la premisa de que las personas establecen relaciones entre ellas y con los otros grupos/comunidades, siendo capaces de construir, preservar, compartir y reflexionar sus conocimientos y experiencias.

En concordancia con Freire:

Consciente de que puedo conocer social e históricamente, sé también que lo que conozco no podría escapar a la continuidad histórica. El saber tiene historicidad. Nunca es, está siempre siendo (...) La historia es tan ir-a-ser, como nosotros (...) como el conocimiento que producimos (...) Sería impensable un mundo donde la experiencia humana se deje fuera de esa continuidad, es decir, fuera de la historia (...) No podemos sobrevivir a la muerte de la historia que, que es hecha por nosotros, nos hace y rehace (Freire, 2001: 18-19).

En este devenir del ser, por lo tanto, los sujetos no pueden ser reducidos a objetos de investigación; tampoco las situaciones en que se encuentran, en las cuales viven, luchan, transforman y producen conocimiento se pueden ver como situaciones inmutables o definitivas, pues el propio ser es inacabado y es sujeto al estar-siendo, al ir-a-ser.

A propósito de ello, en el desarrollo de la investigación, la inserción de los investigadores junto a la comunidad caizara de Playa Mansa y sus alrededores se produjo con una cuidadosa interacción con los habitantes, los cuales autorizaron a través de un Consentimiento Libre e Informado (criterio de rigurosidad ética), la realización de la investigación y la publicación de los datos, alterando sus nombres reales por ficticios, con nombres de peces y animales marinos.

En la Tabla siguiente, se presentan los/as participantes de la investigación: 


\begin{tabular}{|c|c|c|c|c|}
\hline $\begin{array}{c}\text { Nombre } \\
\text { Fictício }\end{array}$ & Sexo & Generación & $\begin{array}{c}\text { Comunidade que } \\
\text { vive }\end{array}$ & Ocupación \\
\hline Robalo & M & A & Portinho & Barquero \\
\hline Salmon & M & A & Playa Mansa & Barquero y Pescador \\
\hline Lenguado & M & A & Playa Mansa & Pescador \\
\hline Raya & F & A & Playa Mansa & Do Lar \\
\hline Sirena & F & A & Playa Castellanos & Dueña de \\
& & & & Restaurante \\
\hline Delfin & M & N & Playa Castellanos & Estudiante \\
\hline Anchoa & F & A & No caiçara & Garzona del \\
& & & & Restaurante \\
\hline Pulpo & M & A & Playa Mansa & Pescador \\
\hline Tiburon & M & N & Playa Mansa & Estudiante \\
\hline Bacalao & M & A & Playa Castellanos & Barquero y Pescador \\
\hline Pez Gato & M & N & Playa Castellanos & Estudiante \\
\hline Congrio & M & N & No caiçara & Estudiante \\
\hline Cangrejo & M & A & Playa Mansa & Pescador \\
\hline Lobo de Mar & M & A & Playa Roja & Pescador \\
\hline Pinguino & M & N & Playa Castellanos & Estudiante \\
\hline
\end{tabular}

Tabla 1. Perfil de informantes claves

Leyenda: M: Masculino; F: Femenino; N: Niño; A: Adulto

Como procedimiento básico en la recolección de los datos se utilizó el registro sistematico de notas en diarios de campo, particularmente en las manifestaciones relacionadas con juegos, luchas, danzas, fiestas, cantos y cuentos de la cultura caizara, los cuales posibilitaron el análisis posterior de las anotaciones. Según Bogdan y Biklen, el diario de campo "es el relato de aquello que el investigador oye, ve, vivencia y piensa en el transcurso de la recogida y reflexiona sobre los datos de un estudio cualitativo" (Bodgan y Biklen, 1994: 150). Además, agregan al respecto de la implementación y construcción del mismo que "las notas de campo consisten en dos tipos de materiales. El primero es descriptivo, donde la preocupación se centra en captar una imagen a través de las palabras del local, personas, acciones y conversas observadas. El segundo es reflexivo, parte que evidencia con mayor densidad el punto de vista del observador, sus ideas y preocupaciones" (Bodgan y Biklen, 1994: 152). 
Después de la lectura de los registros de los diarios de campo, se procedió a la identificación de unidades significativas, las cuales fueron agrupadas en categorías temáticas, organizadas a posteriori en una matriz nomotética de inspiración fenomenológica (Martins y Bicudo, 1989; Martins, 1992; Bicudo y Esposito, 1997; Gonçalves Junior, 2008), formalizando el movimiento intencional en busca de la esencia del fenómeno investigado.

La matriz es una organización formal resultante del tránsito de lo particular a lo general, en el cual se explicita la comprensión de las proposiciones individuales y sus posibles convergencias, divergencias e idiosincrasias (acepción encontrada en sólo una de las descripciones) con las proposiciones de los demás sujetos informantes de la investigación (Martins y Bicudo, 1989; Martins, 1992; Bicudo y Esposito, 1997; Gonçalves Junior, 2008).

\section{RESULTADOS}

Los resultados son presentados en cuatro categorías que son analizadas a partir de la matriz nomotética expuesta a continuación.

\begin{tabular}{|c|c|c|c|c|c|c|c|c|c|c|}
\hline Categorías & I & II & III & IV & $\overline{\mathbf{V}}$ & $\overline{\text { VI }}$ & $\overline{\text { VII }}$ & VIII & $\overline{I X}$ & $\bar{X}$ \\
\hline $\begin{array}{l}\text { A) Desconfianza } \\
\text { aprendida }\end{array}$ & & & 1; 7; & & & $\begin{array}{c}4 ; 7 ; \\
10 d ; \\
16 ; 17 d\end{array}$ & & & 5 & \\
\hline $\begin{array}{l}\text { B) Maritorio } \\
\text { lúdico }\end{array}$ & & 2 & $2 ; 4$ & 3 & $1 ; 4$ & $8 ; 11$ & $\begin{array}{c}2 ; 3 ; \\
4 ; 5 ; \\
6 ; 8\end{array}$ & $\begin{array}{l}1 ; 2 ; \\
3 ; 11 ; \\
13\end{array}$ & 2 & \\
\hline $\begin{array}{l}\text { C) Convivencia } \\
\text { emergente }\end{array}$ & 1 & 1; 3 & $\begin{array}{l}3 ; 5 ; \\
9 ; 10\end{array}$ & $\begin{array}{c}1 ; 2 ; \\
4 ; 5 ; \\
6\end{array}$ & $2 ; 3$ & $\begin{array}{c}1 ; 2 ; \\
3 ; 5 ; \\
6 ; 9 ; \\
12 ; 13 \\
15\end{array}$ & $1 ; 7$ & $5 ; 6 ; 8$ & $\begin{array}{l}1 ; 3 ; \\
4\end{array}$ & 1 \\
\hline $\begin{array}{l}\text { D) Festividad } \\
\text { religiosa }\end{array}$ & & & & & & & & $\begin{array}{l}4 ; 7 ; \\
10 ; 12 ; \\
14\end{array}$ & & \\
\hline
\end{tabular}

Tabla 2. Matriz Nomotética

\subsection{DESCONFIANZA APRENDIDA}

Esta categoría reúne unidades significativas que refieren a comportamientos adoptados por los moradores de la comunidad y, en menor grado, por los propios investigadores. Estos últimos se posicionaron en contra de la explotación turística, que vivenciaron principalmente en el lado urbanizado de la Isla. Más adelante, se exponen trechos de los 
diarios de campo, que muestran al equipo de investigadores/as quienes después de haber negociado un determinado valor con Jiperos (tipo de transporte de la Isla), estos últimos modificaron su precio en el momento que percibieron la posibilidad de mayor ganancia de dinero producto de los barcos cruceros que recalaron en la Isla, transportando al grupo de investigación a la Bahía de Castellanos. Tal situación se explica por la condición de los caminos y su consecuente desgaste de los Jeeps (exigencia del equipo investigador), y en contraste, lo ventajoso y cómodo de realizar paseos con los turistas por las playas del sector urbano cercano.

En el camino para el muelle, o Jipeiro telefoneó al celular de una de las investigadoras para saber lo que habíamos decidido y ella le dice que no se consiguió ir de canoa o barco, ni tampoco con la ayuda del Parque. No viajaríamos para Playa Mansa en aquel momento, pues por una cuestión de principios habíamos decidido no someternos a la colusión ejercida por los Jiperos, quienes habían elevado demasiado el precio del transporte (Diario III, 1-A).

En este sentido, podemos citar dos trechos que demuestran los cuidados y protección demostrados por las personas al relacionarse con nuestro grupo de "extraños". El primero describe una situación acerca de la caipiriña de hoja de mandarina, una bebida típica del local visitado, que dejó al grupo de investigación curioso, por lo siguiente: “(...) comentamos que Anchoa había hablado sobre la caipiriña de hoja de mandarina y Raya expresó que era efectivamente hecha con hojas de mandarina, pero que el modo de extraer el jugo de las hojas era propiamente un secreto" (Diario III, 8-A).

El segundo retrata la conversación de uno de los investigadores con una señora de la comunidad de Playa Roja. En dicha ocasión, el investigador fue en busca de harina de mandioca y coco, siguiendo la orientación de una habitante de la Playa de Castellanos. En esa situación, la señora informó que no había harina producida y cuando el investigador preguntó: “(...) si podía llevar algunos cocos ella respondió que la gente de Castellanos comenta un poco sobre el coco de Playa Roja y muchas personas aparecen por allá pidiendo, y afirmó que estos son sólo para los integrantes de la comunidad” (Diario VI, 4-A).

Los cuidados en su comportamiento, anteriormente citados, son comprendidos como formas de desconfianza aprendida, tienen sus motivaciones explicitadas en las conversaciones que se tuvieron con dos integrantes de la comunidad, quienes se justifican citando experiencias con otros grupos que visitaron la Isla.

(...) un investigador preguntó si podía ver la canoa junto al hermano de ella y si eventualmente podía fotografiarla. Ella respondió que podríamos ver la canoa, pero que para fotografiar o filmar personas deberíamos conversar con más calma, pues ya había acontecido con otros grupos que los fotografiaron, pero que no dieron copias para ellos o algún testimonio de las mismas. (Diario III, 7-A).

Un de los investigadores alertó que el objetivo central de la investigación eran los juegos y la moradora de la comunidad planteó que mucha gente va allá, no pide autorización, no tienen educación y que no la respetan sólo porque ella no tiene mucho estudio (Diario VI, 7-A).

Raya comenzó a ojear el libro de la cultura caizara, elogiando el autor, pero destacaba que no era caizara. Discordó en algunos puntos del libro, como la receta de azul marino (...); la ausencia de identificación de personas presentes en las fotos (detalle que el grupo investigador ya había comentado y cuestionado) y consiguió la foto de una canoa, cuya denominación, en el libro, es de una canoa de boga, pero según ella, ilustra una canoa caizara y no de boga (...) (Diario VI, 16-A). 
En esta categoría también se presentan dos divergencias, demostrando que la desconfianza aprendida inicialmente presentada disminuyó conforme el grupo de investigación fue siendo conocido por las comunidades. Los fragmentos de diarios que siguen muestran cómo situaciones anteriormente citadas se desarrollaron de igual forma, posteriormente, es decir, en forma positiva, en la medida que el conocimiento mutuo aumentaba entre el grupo investigador y los habitantes.

En esta ocasión reveló el secreto de la hoja de mandarina, a saber: se macera un poco más de media taza de hojas de mandarina con un poco de cachaza y azúcar, hasta extraer el jugo de las hojas (eso es lo que da el color anaranjado característico a la bebida), se aumenta la cachaza y el hielo, se puede también colar (Diario VI, 17d-A).

Fuimos bien recibidos por ella y los demás miembros de la comunidad, quienes nos presentaron el lugar donde se realiza la producción de harina de modo artesanal. Después nos mostraron la huerta de la comunidad, el fogón a leña y sus famosos coqueiros, de los cuales tomamos algunos cocos, juntamente con los moradores, para producir una cocada propia en la Playa Mansa (Diario VI, 10d-A).

\subsection{MARITORIO LÚDICO}

Esta categoría emerge desde unidades de significado y extractos que favorecen el conocimiento de algunos juegos practicados por las comunidades caizaras, tanto en la agua de mar (tambien de río), como en la tierra (también en la arena de la playa). Del mismo modo, es singular la generación de un espacio de interface entre mar y tierra, de ahí el neologismo "maritorio"; de igual forma, esta categoría también revela el estar con y entre personas de la comunidad e investigadores, lo que posibilitó diversos momentos de interacción en la realización de los juegos.

Los siguientes extractos de los diarios de campo nos permiten identificar los juegos más practicados en las comunidades observadas.

En un paso por la Playa de Castellanos se observó niños jugando futbol en la arena, siendo los arcos pequeños formados con bambú (Diario III, 4-B).

Durante la conversación (...) le preguntamos qué jugaban los niños en la Playa Mansa y en la región cuando ella era niña. Ella respondió que los varones jugaban mucho al trompo en la tierra, en un lugar un poco separado de la orilla de la playa, y también hacían barquitos, en general, hechos de madera por los padres (...) Y las niñas, dice ella, jugaban "amarillita"5 en la arena de la playa en las noches de luna. Jugaban también al dormir en la playa para esperar a los hermanos que llegaran de la pesca. Cuando ellos llegaban con las canoas corrían detrás de los niños y siempre atrapaban a los más pequeños y terminaban chapoteando en el mar (Diario III, 6-B).

Se observó que los tres niños jugaban a "jipitos y canoas". En el diálogo con ellos, comentaban que jugaban a las canoas y que el juego consistía en una miniatura de canoa y del remolcador, similar al utilizado por sus padres, quienes habían confeccionado las miniaturas. Los niños empujaban la miniatura de canoa con un cordón amarrado a una piedra que

5 Similar al juego avión o luche (dibujo sobre la arena o tierra de una sucesión de cuadros con numeros que asemeja un avión). 
funcionaba como una especia de ancla. Al lado y fuera del agua se encontraban dos jeeps (juguetes) y una especie de cuadri-ciclo para el camino de juego, específicamente para jugar a los "jiperos". Informaron también que comúnmente juegan pega-pega, esconde-esconde, ayuda-ayuda y a chutear el balón. Después del diálogo hicieron demostración del juego de la canoa en un pequeño río llamado Barra (Diario V, 1-B).

En la Playa de Figueira dice ser típico el juego de subir una roca grande por medio de una cuerda y de ella saltar al mar (Diario VIII, 11-B).

Además del gusto por los juegos que se realizan entre las diversas comunidades, también se reveló el gusto por el canto y la danza de ritmos del genero "forró" y "sertanejo"6 a través de la realización de bailes, con sanfoneiros ${ }^{7}$ y guitarristas de la región.

(...) vimos, a través de una ventana de uno de ellos, una guitarra en la pared, por lo que preguntamos si él tocaba. Dijo que sí y que con frecuencia participaban de los bailes realizados en la Playa de Castellanos, donde tocaban canciones sertanejas acompañado de otros guitarristas y sanfoneiros (Diario VI, 11-B).

(...) Anchoa comentó, con dos investigadoras, que todo los domingos acontecía un juego de futbol alli en Castellanos y que venían personas de varias comunidades para jugar, después de la pesca. Al final de la tarde retornamos a Playa Mansa con la invitación de Sirena y Anchoa para volver en la noche al Forró que acontecería en el bar de ellas (Diario VII, 8-B).

Dentro de diversos pasajes de los diarios descritos por los miembros del grupo de investigación en relación a los juegos, destacamos el día en que dos de los participantes de grupo prestan sus bicicletas a los niños del lugar (Tiburón, Delfin y Pinguino).

(...) Sirena llamó a sus hijos (Delfin y Pinguino) que estaban ansiosos por andar en las dos bicicletas de los investigadores. Prestamos las mismas a ellos y enseguida comenzaron a pedalear. Inmediatamente se aproximó a nosotros otro niño, Tiburon (...) los tres quedaron revisando las bicicletas, observados por un integrante del grupo de investigación, que además los orientaba (Diario VII, 4-B).

La forma de organización autónoma de jugar es visible entre los/as niños/as y los adultos, caracterizándose por el cuidado y cooperación, principalmente en la relación de los mayores con los menores durante las prácticas. Asimismo, se evidenció la relación entre géneros. Estos aspectos se pueden apreciar en los extractos expuestos a continuación:

Observamos que ahora había un grupo mayor de niños y niñas experimentado las bicicletas, la mayoría lograba pedalear, pero los menores eran llevados en la parrilla porta equipaje o en el caño del marco por uno de los mayores, en general después de las orienctaciones de algunos miembros del grupo de investigación. Se comentó la facilidad de algunos niños y

$6 \quad$ Forró es un ritmo y danza típicos de la Región Nordeste del Brasil practicada en las fiestas juninas (fiesta originariamente realizada en celebración de la cosecha) y otros eventos. Con la migración de nordestinos en busca de trabajo hacia otras regiones de Brasil, el forró se expandió por el país. Tradicionalmente, esta danza es producida por tríos, compuestos de un sanfoneiro o acordeonista, un tamborilero y un tocador de triángulo. A su vez, en el ritmo sertanejo o caipira (campesino) el sonido de la guitarra es predominante y, en general, cantado por duplas incluyendo primera y segunda voz. Las letras tradicionales acostumbran tener elementos que tratan del cotidiano vivir en el campesino o temas de relaciones amorosas.

7 Dícese de aquel que toca sanfona o acordeón, siendo éste un instrumento musical compuesto por fuelle, diapasón y dos cajas harmónicas de madera. 
niñas para andar en bicicleta a lo que Anchoa comentó que algunos de ellos ya habían tenido bicicletas pero que el rocío marino las daña demasiado (Diario VII, 6-B).

En este juego los investigadores observaron la actitud de los mayores quienes pasaban el balón a los menores, permitiendo una mayor participación de los niños y niñas en el juego (Diario VIII, 2-B)

En el trecho entre la salida del camino hasta el bar de Sirena, dos investigadores observaron siete niños que jugaban "tontito" (...) les llamó la atención la presencia de una niña en el grupo que también participaba del juego (Diario VII, 2-B).

A pesar de la cooperación y la comunión en general observadas por el grupo de investigación, “(...) notamos aún que uno de los niños que estaba en la ronda, la menor del grupo, no recibía el balón" (Diario VII, 3-B). Lo que indica una divergencia en relación las características señaladas mas arriba. La dimensión de tal divergencia no fue posible profundizarla en la experiencia desarrollada en el trabajo de campo realizado.

\subsection{CONVIVENCIA EMERGENTE}

En esta categoría se comprende el proceso de construcción de una convivencia con el otro, donde incluye compartir experiencias, saberes y conocimientos entre los investigadores y los caizaras y vice-versa, un ejemplo que evidencia tal situación es el siguiente trecho del diario de campo V:

Ya en la playa, preguntaron a Lenguado si solamente los dos conseguirían colocar la canoa en el mar. Dos integrantes del grupo de investigación oyeron y preguntaron si podían ayudar, llamaron a los demás miembros de grupo que también estaban en la playa en ese momento. Todos ayudaron a Lenguado a colocar en el mar su canoa, con la cual él transportó a Raya hasta Sombrío para que ella recogiera las autorizaciones de los padres para que los niños/as de la comunidad realizaran un paseo al zoológico de San Pablo días después. Paralelamente, dos pescadores que venían de Castellanos sabían, por Anchoa, que necesitábamos repelente para mosquitos y se ofrecieron para comprar en la ciudad y traer al día siguiente cerca del mediodía. Aceptamos, les dimos el dinero y pedimos que trajeran tres unidades. En la tarde, dos investigadoras buscaron a Raya (mujer caizara) en su casa, solicitando ayuda para una tercera investigadora que estaba con inflamación en los pies debido a las picaduras de insectos (signos de alergia). Raya entró en contacto con el Parque Estatal por radio y dijo que nos informaría posteriormente. En ese intertanto, en la playa, un pescador preguntó a un investigador si podía llevar un poco de agua de los investigadores para sí, el investigador respondió afirmativamente y además ofrecio su ayuda y la del grupo para cualquier otra necesidad que se presentara (Diario V, 3-C).

Hubo momentos en los cuales fue posible notar situaciones de involucramiento en el intercambio de informaciones del grupo de investigación y personas de la comunidad.

Dos investigadores llegaron a la escuela con una bolsa plástica llena de residuos recogidos en el camino. Dijeron que había mucho más por recoger, pero que con el aumento de la lluvia no pudieron más. Cuando la cena estaba casi lista el grupo recibió la visita de Raya (...) ella vino a preguntar si podíamos cargar sus pilas en una de las tomas de energía de la escuela. Obviamente aceptamos y ella realizó lo que precisaba (Diario III, 5-C).

Raya dijo que ya estaba oscuro y tarde y que ella no lo había percibido. Preguntó si alguien tendría una linterna, a lo que una de las investigadoras comentó que Anchoa les había mostrado 
una lata que estaba abandonada en el camino y que era utilizada para ese fin (linterna), por lo que fue llevada a la escuela (Diario III, 10-C).

Con más tiempo de estadía en la localidad, la convivencia entre los investigadores/ as y las personas de la comunidad se acentuó y amplió, tanto en tareas propias de la comunidad, como en el intercambio de información sobre las mismas. Tal situación se puede observar en los siguientes trechos seleccionados.

En seguida todos retornaron para el almuerzo y luego que llegaron al alojamiento, encontraron a parte del grupo que se preparaba para salir hacia el cerco localizado en la Playa de Figueira, con Bacalao, (...) él y los tres del grupo estaban queriendo ir al cerco y como estaba en el lugar de Lenguado, quien estaba en reposo por una lesión en el pie, los invitó a ir con él (Diario VII, 5-C).

(...) De inmediato iniciamos la preparación de la cocada en el alojamiento en que nos encontrábamos, todos fueron ayudando, algunas partían los cocos con un martillo que estaba guardado en la caja de herramientas de la escuela, otros retiraban con cuchillos y cucharas la pulpa de coco de la cascara. Raya, al percibir como hacíamos el proceso, nos corrigió diciendo que sólo podía quedar la parte blanca del coco, es decir, que debíamos raspar bien para no quedar con partes oscuras de la cascara dentro de la cocada (Diario VI, 12-C).

Los cuidados de unos con otros también merece ser destacado, pues aclara más profundamente el sentido de esta categoría y revela la convivencia emergente entre los investigadores y los caizaras y vice-versa.

Cerca de las 19.30, Raya vino a visitarnos, trayendo con ella melisa y jengibre para un té, ambos ingredientes estaban dirigidos, prioritariamente, para un integrante del grupo de investigación que estaba con un poco de dolor de garganta (Diario VI, 13-C).

Llegando al cerco flotante Bacalao se dirigió, justamente con otros dos caizaras que lo acompañaban, en la canoa a remos para la retirada de los peces atrapados en la armadilla y pidió a los investigadores esperar en la canoa con motor, posteriormente ambas canoas volvieron a la Playa Mansa. En ese lugar, caizaras e investigadores recogieron las canoas y descargaron los pescados en cajas plásticas, llevándolos a las cámaras frías. Bacalao abrió la boca de un pez espada mostrando sus dientes, intentando explicar el peligro que corríamos yendo juntos en la canoa menor al cerco flotante. Mostró también el pez cabra, conocido también como pez volador, abrió sus aletas evidenciando el parecido con las asas y que ayudan a planear en sus saltos fuera del agua. Agradecimos la oportunidad, nos despedimos y fuimos a almorzar al alojamiento (Diario VIII, 6-C).

\subsection{FESTIVIDAD RELIGIOSA}

Esta categoría está compuesta por cinco unidades de significado, todas referidas al diario número ocho, y revela detalles de la religiosidad del pueblo caizara. Ellas muestran la confluencia de la religiosidad con la fiesta. En una ocasión, por ejemplo, percibimos personas de diferentes religiones organizándose para participar de la Fiesta de San Sebastián. Tal situación se genera por la posibilidad de encuentro con otras personas, por la celebración misma, independiente, inclusive, de la matriz religiosa personal.

Destacamos que la recolección de datos fue realizada en el mes de enero, época de alta temporada turística, momento en que se presentan las mejores posibilidades de ganancia en el comercio local, incluyendo los bares y restaurantes. Aún así, Anchoa cerró 
su tienda para dirigirse a la fiesta de San Sebastián, como se aprecia en los extractos siguientes: “(...) fueron hasta el bar y restaurante de Sirena, entonces Anchoa comentó que en la tarde se cerraría el bar para ir a San Sebastián, la fiesta del patrono de la ciudad" (Diario VII, 4-D).

Otro evento festivo religioso que congrega muchas personas en Ilhabela es la Fiesta de Reyes Magos:

Raya comentó además, de la Fiesta de Reyes Magos, que acostumbra de salir de la Villa (centro de la Ilhabela) hasta las otras playas, a pie o en barco (Diario VIII, 14-D).

También es posible percibir confluencia de la religiosidad con la fiesta y juegos en el relato de Raya, quien recordando sus tiempos de infancia en las playas de la región, comentó que:

En la Playa Mansa, donde ella vive, cuenta que era común construir un "Judas" con el tronco de un árbol de banana invertido, pues las raíces del árbol parecían el cabello. Además, comúnmente tomaban una chaqueta negra del padre de ella para vestir a la representación de Judas y muchas veces llevaban el muñeco, una vez terminado, a las ventanas de la casa de la abuela y lo dejaban ahí para que cuando la abuela abriera las ventanas en la mañana se asustará. Los niños y niñas disfrutaban mucho de esas travesuras. Posteriormente destrozaban el "Judas" totalmente (Diario VIII, 10-D).

Tal extracto del diário de campo revela que se desarrolla un juego infantil a partir de un personaje bíblico, Judas, cuyo punto alto es la diversión al "asustar" a la abuela, atribuyendo sentido lúdico en convergencia con el religioso.

Raya continua hablando, ahora sobre la Playa Roja:

(...) se dice que hay algunas historias de "miedo" que los más viejos cuentan a las generaciones más jóvenes, dentro de ellas se destaca la leyenda que la playa quedó roja (de ahí su nombre) porque ocurrió una falta de respeto en un viernes santo de pasión, cuando los habitantes de la playa jugaron futbol y después hicieron un asado (Diario VIII, 12-D).

Esto ratifica que el aspecto central de la religiosidad caizara está marcado por procesos afectivos y simbólicos, produciéndose un cruce y confluencia entre aspectos sagrados y profanos, entre riesgo y seguridad, entre alegría-divertimiento y miedo-pecado. De tal manera, la religiosidad se confunde con la fiesta en el sentido más amplio del término, pues, sin perder el carácter sagrado, los elementos profanos se presentan con mucha claridad y relevancia.

\section{CONSIDERACIONES FINALES}

En virtud de las posibilidades reales que se generaron de interacción y convivencia con el pueblo caizara de Ilhabela, consideramos que nuestro objetivo fundamental, que consistía en: caracterizar la etnomotricidad caizara en función de la comprensión de las manifestaciones relacionadas con los juegos, luchas, danzas, fiestas, cantos y cuentos, como los procesos educativos involucrados en sus prácticas situadas y junto a los residentes de la comunidad caizara de la Playa Mansa y alrededores, fue preliminarmente cumplido. Las consideraciones aquí presentadas son resultados de un proceso de aproximación, dado que, durante la investigación, el grupo constató la necesidad de continuidad y profundización 
junto a la referida comunidad para la elaboración de una matriz comprensiva común. Esta misma, de hecho, sólo se construye en la cotidianidad, en una convivencia estable y sólida que se logra en el transcurso del tiempo vivido en común.

Por otra parte, las relaciones en la sociedad actual, fundamentalmente euro-céntrica (para profundizar, Cfr. Dussel 2001; 2005), tienen como plataforma el individualismo, la fragmentación (ser/mundo; cuerpo/mente; carne/espíritu; civilizado/salvaje; sagrado/ profano; ludicidad/seriedad; trabajo/ocio). Esto se traduce en la idea de dominación de la naturaleza y, por consiguiente, de las comunidades que a ellas están más vinculadas, las cuales son, a veces, subvaloradas y tratadas como deficientes o ajenas a un proceso civilizador. Consecuentemente, desde el punto de vista de las comunidades, tal situación se va configurando, en el desarrollo del tiempo, en el aprendizaje de la desconfianza sobre aquello que es externo e impuesto como superior y que proviene del continente y del comportamiento de las personas que visitan la isla.

En ese sentido, percibimos la categoría "Desconfianza aprendida", que expresa el comportamiento de las personas de la comunidad,las cuales, en un principio, expresaban acciones de reserva, distanciamiento, contención. Tal situación que se presentó fue constante en los diferentes habitantes que se conocían o con los que se interactuaba por primera vez. Tal situación fue variando en la medida que se fueron construyendo relaciones de confianza entre la comunidad y los investigadores, posibilitando y generando otras acciones, a saber: disposición para conversar sobre sus juegos, danzas, fiestas, cantos y cuentos, originando así la categoria "Convivencia emergente", caracterizada fundamentalmente por la abertura mútua y por las experiencias contingentes de relaciones simétricas basadas en la mutua aceptación.

Los datos recogidos en la comunidad permitieron la percepción de la multi-dimensionalidad de la motricidad caiçara, basada en la existencia más que en la performance; una etmotricidad que se caracteriza desde el flujo de la vida, como notamos en las categorías "Festividades religiosas" y "Maritorio lúdico", en que lo lúdico penetra lo sagrado y vice-versa. Podemos comprender que la dinámica lúdica presente en el pueblo caizara converge con las características geográficas variadas de la isla, que se co-funden con el mar, el río, la playa y la tierra, constituyéndose un lugar para morar, un lugar para trabajar, un lugar para jugar, un lugar para honrar, un lugar para consagrar, en definitiva, un espacio-temporalidad para co-existir con múltiples formas de vida y diferentes manifestaciones plenas de sentido existencial.

Desde el punto de vista metodológico, y dada la amplitud simbólica expresada en los distintos diarios de campo, el grupo de investigación decidió construir una metodología que permitiera interpretar consistentemente los datos a partir de una "confluencia argumentativa", fundamentada en las distintas miradas que construyen un argumento, desde las personas que están viviendo el proceso y otras que lo experimentan desde una posición más externa. De igual forma, las distintas formaciones profesionales permiten la generación de una visión del fenómeno más compleja y densa, favoreciendo de esta forma una comprensión e interpretación más consistente de las distintas unidades de sentido. Esta experiencia permite establecer dos procesos: en primer lugar, que cualquier esfuerzo interpretativo convoca diferentes y profundos recursos lingüísticos, en el sentido profundo de la palabra, aspecto que se puede lograr con mayor profundidad y pertinencia a través del concurso de diferentes miradas y experiencias sobre un mismo fenómeno; en segundo lugar, que es precisamente en la riqueza del diálogo y permanente intercambio 
y relación entre los investigadores con las personas investigadas, que el proceso indagatorio cualitativo va adquiriendo dimensiones transformativas y de generación de un lenguaje que en sí mismo es situado, y es expresión de una nueva configuración en los dos grupos mencionados. Vale decir, se modifican recíprocamente, en lo que Schütz (1995) llamaba referencia cruzada, por lo cual la mirada de personas que no participan de la experiencia de campo puede favorecer la necesaria suspensión y consistencia de las interpretaciones realizadas.

\section{REFERENCIAS BIBLIOGRÁFICAS}

Adams, C. (2000). As populações caiçaras e o mito do bom selvagem: a necessidade de uma nova abordagem interdisciplinar. Revista de Antropologia, São Paulo, v. 43, n. 1, 144-182.

Almeida, Q. (2005). Usos e costumes praianos. En Diegues, A (org.), Enciclopédia caiçara: História e memória caiçara, Vol IV (pp. 47-60). São Paulo: Hucitec - NUPAUB-CEC/USP.

Bicudo, M., Espósito, V. (1997). Pesquisa qualitativa em educação. São Paulo: UNIMEP.

Bogdan, R., Biklen, S. (1994). Investigação qualitativa em educação: uma introdução à teoria e aos métodos. Porto: Porto Editora.

Ministério da Educação e do Desporto-Secretaria de Educação Fundamental, Governo Federal do Brasil (1997). Parâmetros Curriculares Nacionais: Educação Física. Brasília: MEC / SEF.

Campos, S. (2008). Ser caiçara em Ilhabela: as construções de identidades nas tensões entre o passado e o presente. Dissertação (Mestrado em Educação). São Carlos: UFSCar.

Corrêa, F. (1981). A congada de Ilhabela na festa de São Benedito. São Paulo: Escola do Folclore, Livramento.

Diegues, C. (2005). Esboço de história ecológica e social caiçara. En C. Diegues (org.), Enciclopédia caiçara - história e memória caiçara (pp. 273-319). São Paulo: editora Hucitec.

Dussel, E. (2005). Transmodernidad e interculturalidad (interpretación desde la filosofia de la libertación). México City: UAM.

Fiori, M. (1986). Conscientização e educação. Educação e Realidade, vol. 11, n. 1, 3-10.

Freire, P. (2005). Pedagogia da esperança: um reencontro com a pedagogia do oprimido. $12^{\mathrm{a}} \mathrm{ed}$. Rio de Janeiro: Paz e Terra.

Freire, P. (2001). A sombra desta mangueira. São Paulo: Olho d'água.

Gonçalves, L. (2007). A motricidade humana no ensino fundamental. En I Seminário Internacional de Motricidade Humana: passado-presente-futuro (pp. 29-35). São Paulo: ALESP.

Gonçalves, L. (2008). Lazer e trabalho: a perspectiva dos líderes das centrais sindicais do Brasil e de Portugal em tempos de globalização. En L. Gonçalves (org.), Interfaces do lazer: educação, trabalho e urbanização (pp. 54-108). São Paulo: Casa do Novo Autor.

Gonçalves, L. (2010). Etnomotricidade: multiculturalismo e educação física escolar. En D. Carreira Filho y W. Correia (Org.), Educação física escolar: docência e cotidiano (pp. 49-67). Curitiba: CRV.

Martins, J. y Machado, O. (1997). Introdução ao curso "seminários avançados em fenomenologia". En M. Bicudo y V. Espósito (org.), Joel Martins... um seminário avançado em fenomenología (pp. 17-28). São Paulo: EDUC.

Martins, J. (1992). Um enfoque fenomenológico do currículo: educação como poíesis. São Paulo: Cortez.

Martins, J. y Bicudo, M. (1989). A pesquisa qualitativa em psicologia: fundamentos e recursos básicos. São Paulo: Moraes/EDUC.

Merleau-Ponty, M. (1996). Fenomenologia da percepção. $2^{\mathrm{a} e d . ~ S a ̃ o ~ P a u l o: ~ M a r t i n s ~ F o n t e s . ~}$ 
Sérgio, M. (1999). A racionalidade epistémica na educação física do século XX. En M. Sérgio et al., O sentido e a acção (pp. 11-30). Lisboa: Instituto Piaget.

Sérgio, M. (1994). Motricidade humana: contribuições para um paradigma emergente. Lisboa: Instituto Piaget.

Silva, T. (1991). Sentido dos existenciais básicos para Heidegger. Dissertação. (Mestrado em Educação). São Paulo: PUC/SP.

Schutz, A. (1995). Fenomenología del mundo social. Buenos Aires: Paidos.

Villela, A. (2003). Favelização no litoral norte: um modelo de turismo e seu impacto na estrutura urbana. Monografia (Graduação em Arquitetura). São Paulo: USP. 